\title{
Dynamic Measurement of Temperature, Velocity, and Density in Hot Jets Using Rayleigh Scattering
}

\author{
Amy F. Mielke ${ }^{1}$, Kristie A. Elam ${ }^{2}$ \\ 1: Optical Instrumentation and NDE Branch, NASA Glenn Research Center, Cleveland, OH, USA, \\ Amy.F.Mielke@nasa.gov \\ 2: Jacobs Sverdrup, Cleveland, OH, USA, Kristie.A.Elam@nasa.gov
}

\begin{abstract}
A molecular Rayleigh scattering technique was utilized to measure time-resolved gas temperature, velocity, and density in unseeded gas flows at sampling rates up to $10 \mathrm{kHz}$. A high power continuous-wave (cw) laser beam was focused at a point in an air flow field and Rayleigh scattered light was collected and fiber-optically transmitted to a Fabry-Perot interferometer for spectral analysis. Photomultipler tubes operated in the photon counting mode allowed high frequency sampling of the total signal level and the circular interference pattern to provide time-resolved density, temperature, and velocity measurements. Mean and rms velocity and temperature, as well as power spectral density calculations, are presented for measurements in a hydrogen-combustor heated jet facility with a 50.8-mm diameter nozzle at the NASA Glenn Research Center (GRC). The Rayleigh measurements are compared with particle image velocimetry data and CFD predictions. This technique is aimed at aeronautics research related to identifying noise sources in free jets, as well as applications in supersonic and hypersonic flows where measurement of flow properties, including mass flux, is required in the presence of shocks and ionization occurrence.
\end{abstract}

\section{Introduction}

A dynamic Rayleigh scattering technique has been developed to address the need for a nonintrusive, high frequency response multiple-property flow measurement technique. The current state-of-the-art for non-intrusive temperature measurement, specifically in cases where the local pressure is unknown, is limited to sampling rates less than a few $\mathrm{kHz}$. Additionally, there are no techniques that can provide non-intrusive time-resolved measurement of gas temperature, velocity, and density simultaneously at rates beyond a couple $\mathrm{kHz}$. Molecular scattering techniques are capable of providing gas temperature and density information since the gas molecule properties are directly determined. Eckbreth (1996) provides the details of several molecular scattering based techniques, such as Coherent Anti-Stokes Raman Spectroscopy (CARS), spontaneous Raman and Rayleigh scattering, and Laser Induced Fluorescence (LIF), with an emphasis on their use in combustion applications. Other molecular-based techniques include laser-induced gratings (Cummings 1995, Hart et al. 1999), filtered Rayleigh scattering (Boguszko and Elliott 2005), collective light scattering (Bonnet et al. 1995), and molecular flow tagging techniques (Koochesfahani 1999). The temporal response of most optical measurement techniques is typically limited by the repetition rate of pulsed lasers; usually on the order of 10-100 Hz. Advances in laser and camera technology have led to recent developments of temporally resolved velocity measurements using Particle Image Velocimetry (PIV) at rates exceeding $10 \mathrm{kHz}$ (Wernet 2007). Although high repetition rate lasers exist, they do not have the narrow linewidth, stable frequency, and high beam quality typically needed in spectroscopic Rayleigh scattering flow diagnostics. The presented molecular scattering technique can measure density, temperature and velocity simultaneously by collecting Rayleigh scattered light from a high power single-frequency cw laser beam, routing the optical signal via fiber optics, and spectrally filtering the light using a Fabry-Perot interferometer (FPI). The signal strength and spectrum of the light contain information about the density, temperature, and velocity of the flow. Photomultiplier tubes (PMTs) are used in a novel 
configuration to detect the signal strength and interferometric fringe intensity pattern. Sampling the number of photons collected during very short integration periods provides time-resolved pointwise multi-property measurements with spatial resolution of approximately $0.1 \mathrm{x} 0.1 \mathrm{x} 1 \mathrm{~mm}^{3}$ at rates up to $10 \mathrm{kHz}$. The ability to obtain dynamic high frequency response measurements of multiple properties simultaneously is a valuable tool for compressible, turbulent flow research. Dynamic flow measurements are particularly useful to aeroacoustics researchers who are interested in correlating flow property fluctuations with far field acoustic fluctuations, leading to validation and improvement of computational aeroacoustics (CAA) noise prediction codes. Knowledge of velocity-temperature fluctuation correlations is also needed to improve computational fluid dynamic (CFD) models of compressible turbulent flows.

Previous works using molecular Rayleigh scattering to make temperature, velocity, and number density measurements in various environments (Lock et al. 1992, Panda \& Seasholtz 1999, Mielke et al. 2005), and dynamic density and velocity measurements in supersonic free jets (Seasholtz et al. 2002) have been reported. The technique described in this paper has been demonstrated previously in various lab-scale flow studies and validated by comparison with hotwire and coldwire probe measurements (Mielke 2008). This paper describes the application of the technique for characterization of heated air flows in a large-scale test facility. In response to NASA's mission to reduce aircraft noise, experiments have been conducted to evaluate temperature fluctuations that could act as a potential additional source of jet noise. Measurements are performed using the Rayleigh scattering technique in the Small Hot Jet Acoustic Rig (SHJAR), which is a heated nozzle facility located in the AeroAcoustic Propulsion Laboratory (AAPL) at the NASA Glenn Research Center (GRC). This facility can provide up to Mach 2 flow conditions with maximum temperatures approaching $970 \mathrm{~K}$. Data acquired in this facility at subsonic heated flow conditions using a 50.8$\mathrm{mm}$ diameter convergent nozzle are compared with temporally resolved PIV (TR-PIV) and conventional PIV measurements and computational fluid dynamics (CFD) predictions. While all three flow parameters (density, temperature, and velocity) have been measured in this experiment, only velocity and temperature results are presented here since density measurements using Rayleigh scattering in this facility were previously reported (Panda et al. 2004).

\section{Rayleigh scattering and spectral analysis}

In molecular Rayleigh scattering an incident electric field interacts with an atom or molecule inducing a dipole moment that oscillates and radiates at the frequency of the incident field. It is considered an elastic scattering process because the internal energy of the molecule is unchanged and the frequency of the light is changed only by the Doppler effect due to the thermal as well as the bulk motion of the molecules. The frequency spectrum of the scattered light contains information about the gas density, bulk velocity, and temperature. Figure 1 shows a Rayleigh scattering spectrum containing the narrow laser line and a typical Rayleigh spectral peak to illustrate how the flow property measurements are obtained from the spectral information. If the gas composition is fixed, the total intensity of the Rayleigh scattered light is directly proportional to the gas density $\rho$. The width of the spectrum is related to the gas static temperature $T_{s t}$. The frequency shift between the laser peak and the Rayleigh peak is proportional to a single component of the bulk flow velocity. The measured velocity component, $u_{k}$, is in the same direction as the interaction wave vector $\mathbf{K}$, which is the bisector of the incident and scattered light wave vectors, $\mathbf{k}_{\mathbf{0}}$ and $\mathbf{k}_{\mathbf{s}}$, respectively, that are separated by the scattering angle $\chi_{s}$ (Fig. 2). The interaction wave vector and its magnitude $K$ are given by:

$$
\mathbf{K}=\mathbf{k}_{\mathrm{s}}-\mathbf{k}_{\mathbf{0}}
$$




$$
K=|\mathbf{K}|=\frac{4 \pi}{\lambda}\left(\sin \frac{\chi_{s}}{2}\right)
$$

The geometry of the optical arrangement in an experiment is designed such that the desired component of the velocity vector $\mathbf{v}$ is measured:

$$
u_{k}=\frac{\mathbf{K} \cdot \mathbf{v}}{K}
$$

Experiments are typically arranged such that the electric field vector $\mathbf{E}$ is perpendicular to the scattering plane defined by the incident and scattered light wave vectors $\left(\beta=90^{\circ}\right.$, 's'-type polarization (Fig. 2)). In this situation the scattering strength is independent of the scattering angle.

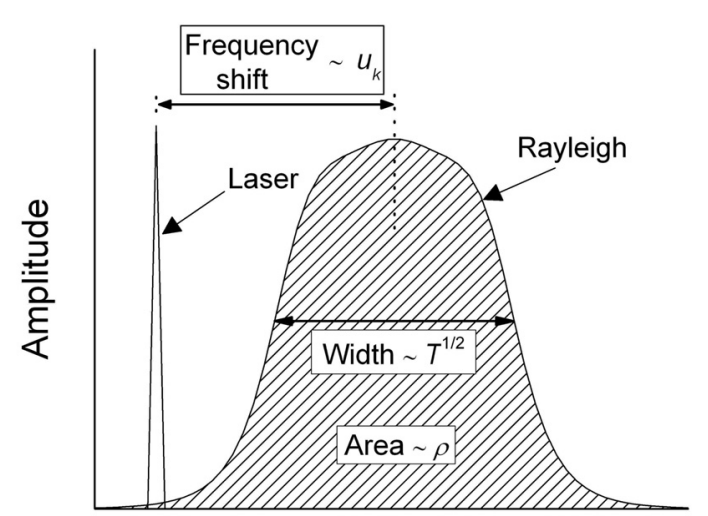

Frequency

Fig. 1 Rayleigh scattering spectrum.

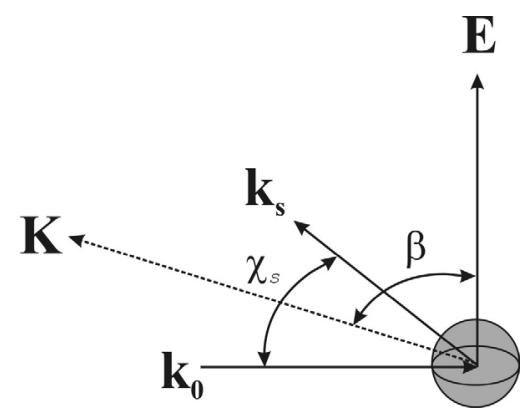

Fig. 2 Light scattering from a moving particle.

Rayleigh scattered light from a defined probe volume is collected into a multimode optical fiber. In this experiment the incident laser beam with power $P_{0}$ is focused to a diameter smaller than the imaged field size, such that the power collected is proportional to the length of the probe volume set by the field size of the collection optics. The Rayleigh light scattered into solid angle $\Omega$ from a probe volume of length $L_{x}$ expressed in terms of expected photoelectron counts is:

$$
\left\langle N_{R}\right\rangle=\frac{\varepsilon P_{0} n L_{x} \lambda \Omega \Delta t}{h c}\left(\frac{d \sigma}{d \Omega}\right) \sin ^{2} \beta
$$

where the overall system efficiency $\varepsilon$ includes detector quantum efficiency and other losses, $n$ is molecular number density, $\lambda$ is the laser wavelength, $\Delta t$ is the integration time over which photoelectrons are counted, $h$ is Planck's constant, $c$ is the speed of light, and $\frac{d \sigma}{d \Omega}$ is the differential scattering cross-section.

The spectrum of the Rayleigh scattered light exiting the optical fiber is analyzed using a planar mirror FPI (Vaughan 1989). A diagram of the FPI system used in this experiment is shown in Fig. 3. The Fabry Perot etalon consists of two parallel planar mirrors. The FPI is operated in the static imaging mode in which the plate spacing $d$ and medium refractive index $\mu$ are held constant while the angle of the incoming light ray $\theta$ is varied by imaging points off-axis. A collimating and 
focusing lens at the input and output of the etalon images the light source (fiber face) at the image plane where a segmented mirror directs regions of the image to photon detectors. The interference fringe pattern is equivalent to the intensity of the image of the fiber face without the FPI modified by the transmission properties of the FPI. The instrument function of the FPI is the Airy function. Imaging a single-frequency light source with a very narrow linewidth (i.e., laser light) through the FPI produces a concentric ring Airy pattern. For spectrally broadened Rayleigh scattered light, the fringes broadened accordingly; the linewidth of the fringes provids a measure of temperature. The frequency shift of the light associated with the bulk flow velocity is determined by a spatial shift in the fringe positions in the image. Due to the finite diameter of the optical fiber source only the innermost fringe is imaged. Figure 4 shows a typical thermally broadened fringe image illustrating how the interference pattern is split up into one circular and three annular regions. The light from each of these regions is detected by four PMTs.

The fringe intensity pattern is a function of both the Rayleigh spectrum and the Fabry-Perot instrument function. The Fabry-Perot instrument function $I_{F P}$ is a function of radial position in the image plane $r_{i}$ and normalized frequency $x_{f}$ which is defined as:

$$
x_{f}=\frac{2 \pi\left(f-f_{0}\right)}{K a}
$$

where $f$ is the frequency of the scattered light and $f_{0}$ is the frequency of the incident laser light. The expected amount of energy collected from the $q^{\text {th }}$ annular region of the interference pattern in terms of photoelectron counts, can be expressed by the following model function:

$$
\left\langle N_{q}\right\rangle=\frac{\left\langle N_{R}\right\rangle}{\pi r_{\max }^{2}} 2 \pi \int_{r_{i, q}}^{r_{o, q}} \int_{-\infty}^{\infty} S_{R}\left(x_{f}\right) I_{F P}\left(x_{f}, r_{i}\right) r_{i} d x_{f} d r_{i}
$$

where $r_{\text {max }}$ is the radius of image of the fiber face, $r_{i, q}$ and $r_{o, q}$ are the inner and outer radii of the $q^{\text {th }}$ annular region, respectively, and $S_{R}$ is the Rayleigh spectrum. Various Rayleigh spectral models are available for use in Eq. (6). The model chosen for this work is a kinetic theory model that was developed by Professor G. Tenti (TENTI S6: 6-moment Rayleigh scattering model) which provides the Rayleigh-Brillouin spectrum for diatomic gases (Boley et al. 1972, Tenti et al. 1974) and is applicable over the full range of expected flow regimes. Professor Tenti has provided his computer code which has been incorporated into the model function given by Eq. (6). Maximum likelihood estimation (MLE) analysis of the experimental data using this developed model function provides estimates of the unknown flow parameters.

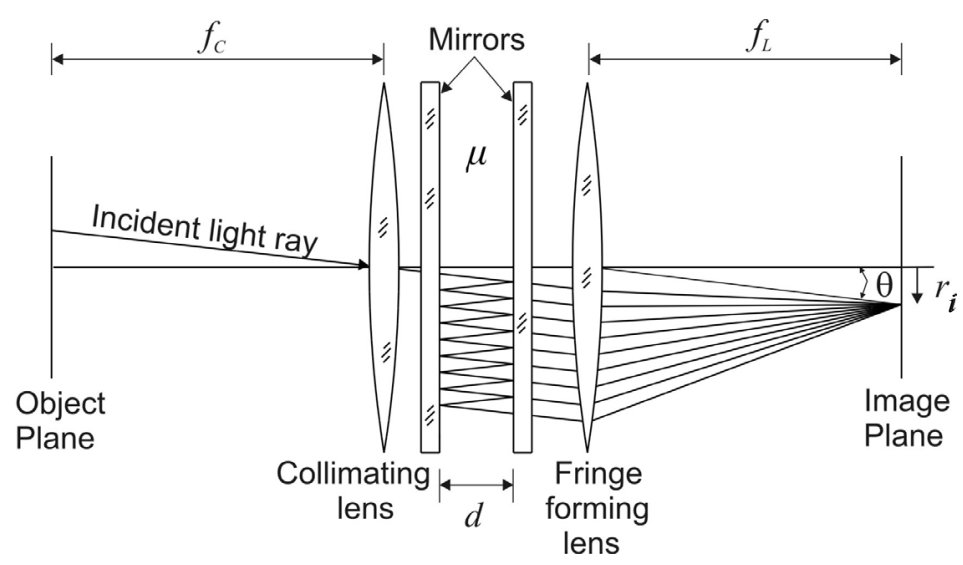

Fig. 3 Fabry-Perot interferometer.

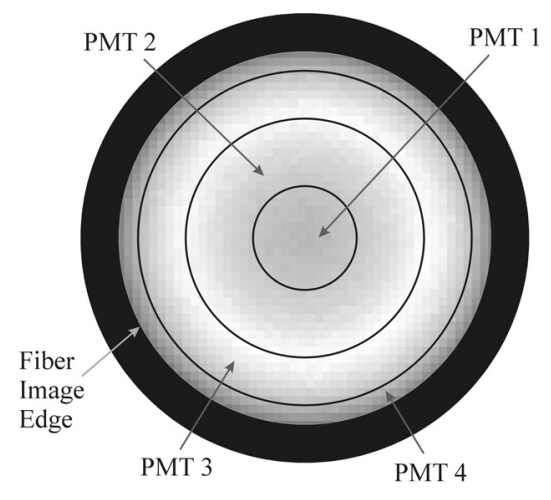

Fig. 4 Dissection of Fabry-Perot fringe pattern into one circular and three annular regions. 


\section{Experiment}

\subsection{Optical setup}

The Rayleigh scattering diagnostic was applied in the Small Hot Jet Acoustic Rig (SHJAR) in NASA Glenn's AeroAcoustic Propulsion Laboratory (AAPL). The SHJAR is a vitiated air heated jet rig that can cover the range of Mach numbers up to Mach 2, and static temperature ratios (the ratio of jet exit temperature to ambient temperature) up to 2.8 using a hydrogen combustor and central air compressor facilities. The AAPL is a $19.8-\mathrm{m}$ radius geodesic dome with its interior covered by sound absorbent wedges that provide the anechoic environment required to study propulsion noise from the SHJAR. The nozzle used in this test was one of a family of convergent nozzles, called the Simple Metal Chevron (SMC) nozzles. The baseline nozzle (SMC000), which has a 50.8-mm exit diameter and a smooth round exit (no chevrons), was tested. A variety of conditions with acoustic Mach number (Ma), which is defined as the ideally expanded velocity divided by the ambient sound speed, ranging from 0.5 to 1.59 and static temperature ratio (TR) from 0.835 to 2.7 were covered in the test matrix; however results from only two exit flow conditions are presented here. These conditions are $\mathrm{Ma}=0.5, \mathrm{TR}=1.765$ and $\mathrm{Ma}=0.9, \mathrm{TR}=2.7$. Measurements were acquired at several axial locations of $2 \leq x / D \leq 20$ and radial locations of $0.0 \leq$ $r / D \leq 1.5$.

A schematic of the laser and collection optics portion of the Rayleigh scattering system around the SHJAR are shown in Fig. 5 and a photograph of the system is shown in Fig. 6. A co-flow system was built specifically for this test (components are shown in blue in Fig. 5) to provide a low speed particulate-free air stream around the jet core. Room air was filtered by a blower system equipped with submicron HEPA filters and was routed to the settling chamber via two $200-\mathrm{mm}$ flex duct sections and exhausted around the nozzle through a straight $250-\mathrm{m}$ long section of $305-\mathrm{mm}$ diameter pipe. A screen was positioned between the settling chamber and the straight exhausting duct section to break up turbulence and straighten the flow. The top view of the facility shown in Fig. 5 illustrates the laser beam propagation and scattered light collection. The laser head of a 10W, 532-nm wavelength, single-frequency, cw laser was located about 1-m below the scattering plane. The vertical black box shown to the right of the nozzle in Fig. 6 housed two turning mirrors, a polarizer, and the focusing lens that routed the beam upwards (out of the plane of the page in Fig. 5) and through the centerline of the jet flow. The laser beam was focused with a $500-\mathrm{mm}$ focal length lens (L1) to a $140-\mu \mathrm{m} 1 / \mathrm{e}^{2}$ diameter at the probe volume. The beam was oriented in the horizontal plane, at a $45^{\circ}$ angle to the primary flow direction. Light was collected at a $90^{\circ}$ scattering angle, collimated and focused by a pair of $f / 3450-\mathrm{mm}$ focal length lenses (L2 \& L3), then re-imaged by another pair of lenses having focal lengths of 150-mm and 100-mm (L4 \& L5, respectively) onto a $0.55-\mathrm{mm}$ diameter multimode optical fiber. The train of lenses provided 1:1.5 imaging and a probe volume length along the laser beam propagation direction of $0.825-\mathrm{mm}$. The incident and scattering wave vectors were arranged such that the axial component of the jet velocity $u$ was measured, as indicated by the $\mathbf{K}$ vector (shown in red) in Fig. 5. The laser and optics were all mounted on a large $\mathrm{x}-\mathrm{y}$ traversing system (not shown in the figures) so that the probe volume could be positioned at various locations in the jet flow.

The 75-m long, 0.55-mm diameter multimode optical fiber transmitted the Rayleigh signal to the sensitive spectral analysis equipment, which was located in an adjacent building and is shown schematically in Fig. 7. The light exiting the fiber was collimated by an $80-\mathrm{mm}$ focal length $f / 1.6$ lens (L6) and was directed through the planar mirror FPI. The FPI had 70-mm diameter mirrors with $80 \%$ reflectivity, $8.7 \mathrm{GHz}$ free spectral range, and reflective finesse of approximately 15 . The Rayleigh scattered light exiting the FPI was focused by a fringe forming lens (L7) having an 
effective focal length of approximately $2700-\mathrm{mm}$, which provided an 18.6-mm diameter image of the fiber face. Light from circular and annular sections of the image were directed toward PMTs by a concentric elliptical mirror system. A photograph of the 'image dissector' is shown in the upper left corner of Fig. 7. The mirrors were machined from 6061 aluminum and the $12^{\circ}$ elliptical surfaces were diamond polished to a mirror finish with about $90 \%$ reflectivity. The mirror diameters, from innermost to outermost, were 5-mm, 12- $\mathrm{mm}, 16-\mathrm{mm}$, and 25-mm. Each mirror sent the respective portion of the fringe image toward PMTs 1, 2, 3, and 4, as shown previously in Fig. 4; the fringe regions are also illustrated near the corresponding PMT in Fig. 7.

The PMTs were operated in the photon counting mode and a PC-based data acquisition system was used to record the signals from the PMTs. Typical photoelectron count rates for this work were on the order of $1 \mathrm{MHz}$. Each signal channel was digitized for 7.98 seconds at $10 \mathrm{kHz}$ sampling rate. The intensity information from the Fabry-Perot fringe sampling was used to obtain temperature and velocity measurements. The density measurement was much simpler, requiring only an overall intensity measurement. A beamsplitter located just in front of the FPI input (Fig. 7) directed approximately $10 \%$ of the incoming Rayleigh scattered light to a lens (L12), which focused the light at PMT 5 for the density measurement. The photoelectron counts recorded by PMT 5 were linearly related to density, which provided an independent yet simultaneous measurement of density along with the velocity and temperature measurements from spectroscopic analysis.

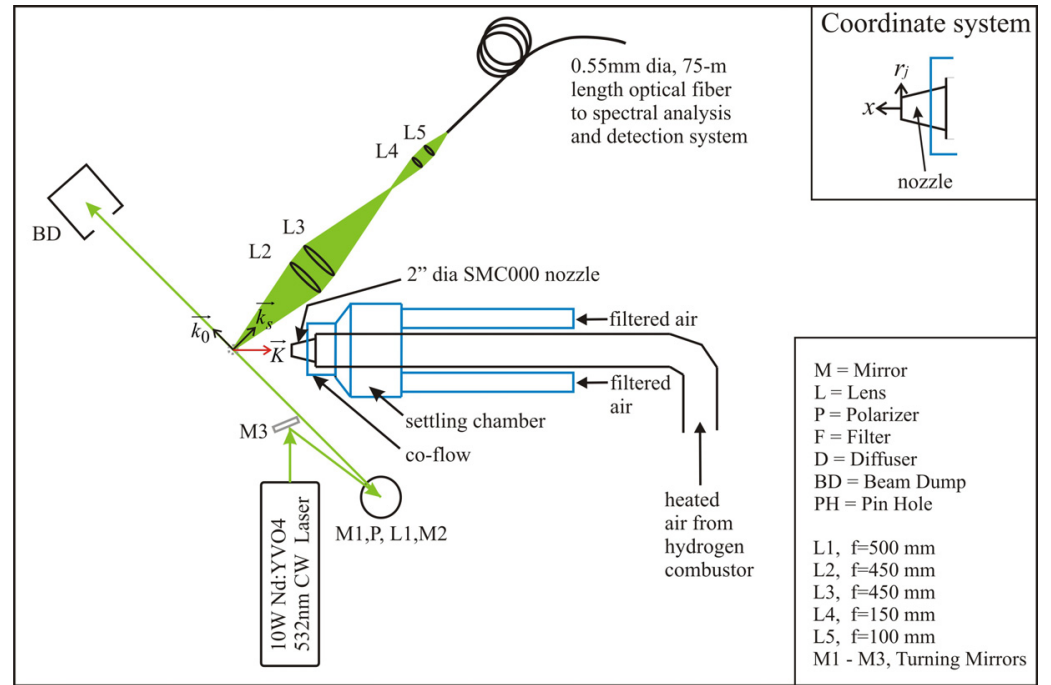

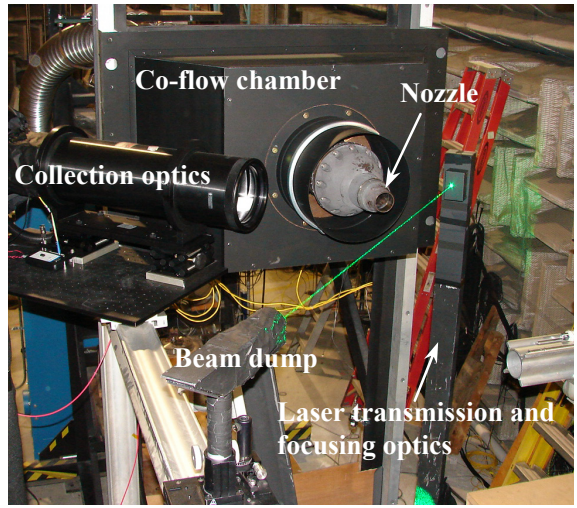

Fig. 6 Photo of the laser and collection optics portion of the Rayleigh system in the SHJAR facility.

Fig. 5 Schematic of laser and collection optics in the SHJAR facility.

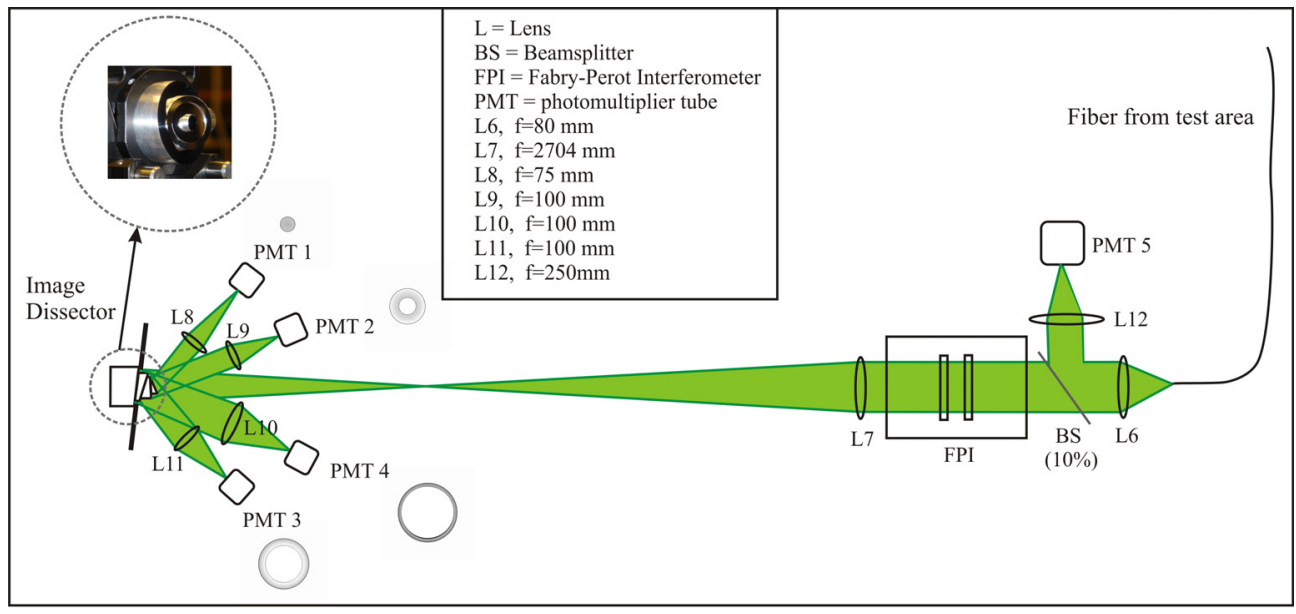

Fig. 7 Schematic of spectral analysis and detection optics in AAPL control room. 


\subsection{Experiment calibration and data analysis method}

For calibration purposes, temperature, density, and velocity measurements were acquired in a steady laminar flow in the jet core over a static temperature range of 239 to $760 \mathrm{~K}$ and a velocity range of 167 to $302 \mathrm{~m} / \mathrm{s}$. The flow was subsonic for all calibration points; therefore the velocity, static temperature, and static density were calculated assuming ideal expansion of an ideal gas. The lowering of specific heat ratio with increasing temperature was taken into account in these calculations. Using this information the linear relationship between PMT 5 photon counts and gas density was established. Also, several unknown parameters, such as system detection efficiencies, effective finesse of the FPI, and fringe forming lens focal length, were evaluated by MLE analysis of the mean photon count data using the ideally expanded flow properties as known values for temperature, velocity, density, and pressure in the model function. Once determined, these parameters were substituted into the model function for use in MLE analysis of the PMT counts for temperature and velocity measurements.

Following calibration, data was acquired at various radial and axial locations and jet exit conditions. Using the linear relation between PMT 5 photon counts and density, and MLE analysis of the photon counts from PMTs 1-4, time histories of instantaneous density, temperature, and velocity were evaluated. The density measured by PMT 5 and the temperature estimate from MLE analysis were used to determine the local pressure using the ideal gas law. Temporally-resolved velocity data previously acquire using TR-PIV at $10 \mathrm{kHz}$ data rate and conventional $10 \mathrm{~Hz}$ PIV data were compared to the Rayleigh data (Wernet 2007, Bridges and Wernet 2003). Reynolds-Averaged Navier-Stokes (RANS) CFD predictions from the work of Khavaran and Kenzakowski (2007a, 2007b) were also compared with mean and rms velocity and temperature values estimated by the Rayleigh technique.

The mean velocity, temperature, and density were calculated and subtracted from their respective time history records to provide zero-mean records, which were used to calculate the power spectral densities of the property fluctuations. Because of the shot noise in the PMT signals, it was necessary to use relatively long data records and calculate power spectra using a technique known as the Welch method of modified periodograms (Welch 1967). In the Welch method, a data record sampled at a rate $f_{s}=10 \mathrm{kHz}$ was subdivided into smaller records of length $L=1024$ samples, which were overlapped by $50 \%$. The modified periodograms of each sub-record were calculated using a Welch data window. These individual periodograms were then averaged to obtain the estimate of the power spectrum and divided by the frequency resolution $\left(f_{s} / L=9.76 \mathrm{~Hz}\right)$ to obtain the power spectral density (PSD). The number of individual periodograms used to calculate the averaged PSD depended on the length of the record used. The 7.98 second data records resulted in 154 overlapped segments. Overlapping the segments by $50 \%$ provided a near maximum reduction in the variance in the spectral estimate (Welch 1967). The resulting PSDs provided fluctuation information up to half of the sampling frequency, and the integral of the PSD was equivalent to the mean square fluctuations. The PSDs were smoothed using a five-point moving average filter.

A common numerical processing method used to eliminate noise from power spectra involves simply subtracting the noise floor from the spectra. Shot noise is broadband noise that contributes equally over all frequencies; therefore it causes a constant offset in the spectrum. If the spectrum flattens out at high frequencies due to lack of significant fluctuations, then the average value of this noise floor can be estimated and subtracted from every point in the spectrum, thereby eliminating the broadband shot noise contribution. This procedure was used to eliminate shot noise in the PSD and rms turbulence estimates; the noise floor was estimated from the last $500 \mathrm{~Hz}$ of the power spectrum. 


\section{Results and discussion}

The SHJAR was operated at jet exit conditions of $U_{j}=167 \mathrm{~m} / \mathrm{s}$ and $T_{j}=495 \mathrm{~K}(\mathrm{Ma}=0.5$, TR $=$ 1.765: set point 23$)$ and $U_{j}=302 \mathrm{~m} / \mathrm{s}$ and $T_{j}=760 \mathrm{~K}(\mathrm{Ma}=0.9, \mathrm{TR}=2.7$ : set point 46$)$ while the Rayleigh probe volume was scanned horizontally from the jet centerline $(r / D=0)$ outward through the shear layer at axial locations of $x / D=2,3,5,6,7,10,14$, and 20. TR-PIV data were acquired at the lower temperature flow conditions (set point 23) in the same facility prior to this test (Wernet 2007) and are included here for comparison with the Rayleigh measurements. Conventional $10 \mathrm{~Hz}$ PIV data were available at set point 46 flow conditions (Bridges and Wernet 2003). Both the nozzle flow and ambient air were seeded in the PIV work to achieve velocity measurements in the core as well as the mixing layer. The hot nozzle flow was seeded with $0.5 \mu \mathrm{m}$ diameter alumina powder, while the ambient fluid was seeded with $0.3 \mu \mathrm{m}$ mineral oil droplets produced by a commercial smoke generator. Reynolds-Averaged Navier-Stokes (RANS) CFD predictions are also compared with the Rayleigh and PIV data. The CFD predictions use a specialized RANS solver by CRAFT (Kenzakowski 2006) that predicts the total enthalpy variance and its dissipation rate in addition to the usual mean and turbulent parameters. The model, in concept, is based on the work of Nagano and Kim (1988) that utilized the internal energy variance, coupled with the standard two-equation turbulence model to predict thermal diffusivity in hot turbulent jets. RANS predictions reported here are part of a larger matrix of set points examined by Khavaran and Kenzakowski (2007a, 2007b), and used as input in jet noise source modeling in heated jets.

Figure 8 shows a radial profile of RANS CFD, Rayleigh, and TR-PIV rms axial velocity, $u$ ', estimates normalized by the jet exit velocity, expressed as percentage, at an axial location of $x / D=$ 3 for set point 23. The Rayleigh and TR-PIV data were both acquired at $10 \mathrm{kHz}$ sampling rate. This plot illustrates the spatial resolution of the various data sources. The resolution of the TR-PIV data was $0.05 \mathrm{D}$ in both the axial and radial directions, with a $0.15 \mathrm{D}$ gap in the radial direction between merged data sets. The conventional $10 \mathrm{~Hz}$ PIV data, which is not represented in Fig. 8, had spatial resolution of $0.023 \mathrm{D}$ in both directions. The Rayleigh data was only acquired at the $x / D$ locations noted above and did not have a regularly spaced grid of points in the radial direction; hence the spatial resolution of the data varied throughout the flow field. A map of the measurement locations for the Rayleigh technique is shown in Fig. 9. There were more radial measurement locations for set point 23 than set point 46 .

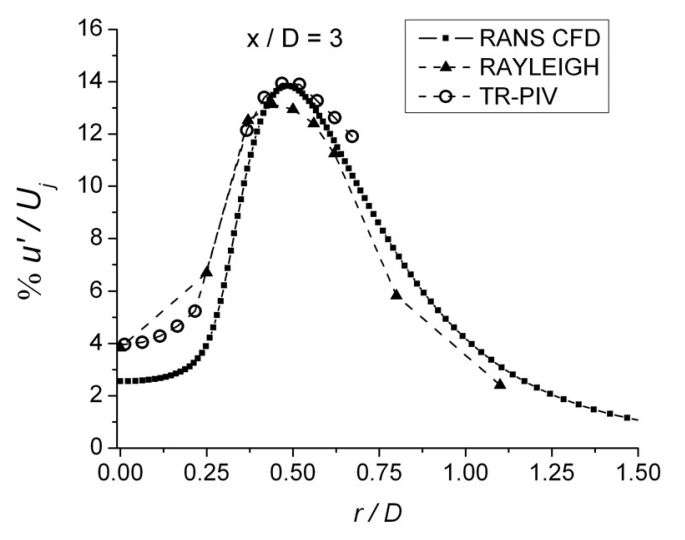

Fig. 8 Radial profile of normalized rms axial velocity fluctuations at $x / D=3$ based on Rayleigh and TR-PIV measurements and RANS CFD prediction for set point 23 .

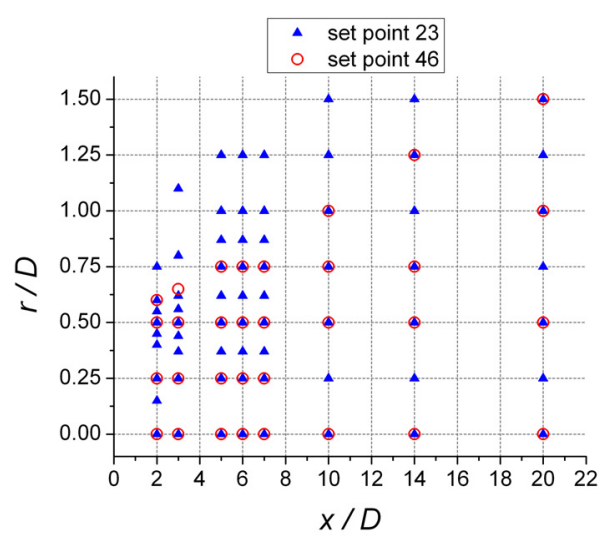

Fig. 9 Map of Rayleigh measurement locations for set points 23 and 46 . 
The rms turbulence for the Rayleigh and TR-PIV data are determined from the integral of the PSD curve as discussed in the previous section. Rayleigh and TR-PIV velocity PSDs are shown for a radial location of $r / D=0.5$ and axial locations of $x / D=3$ and 6 in Figs. 10a and 10b, respectively, for set point 23. The shot noise floor has been subtracted from the Rayleigh PSDs; the TR-PIV technique was not plagued by signal noise in the PSD estimates. The CFD predictions do not provide time-history records and therefore cannot be used to calculate PSD estimates. Also, the low time resolution of the $10 \mathrm{~Hz}$ PIV measurements prohibits the calculation of power spectra. Notice that the Rayleigh PSD predicts slightly lower energy fluctuations than the TR-PIV estimate for frequencies greater than $500 \mathrm{~Hz}$ at $x / D=3$ (Fig. 10a), but the two techniques compare quite well at $x / D=6$ (Fig. 10b). Obtaining accurate PSD and rms fluctuation estimates from the Rayleigh data relies on two assumptions: 1. The noise floor is sufficiently represented by the average spectral value over the last $500 \mathrm{~Hz}$, and 2. Any energy in the spectrum that falls below the noise floor level does not make a significant contribution to the rms fluctuations. If these two assumptions are not met, the noise floor subtraction technique may cancel out energy that is not purely noise. This tends to be a problem in regions where higher frequency fluctuations are expected since the spectrum is less likely to have reached the noise floor level by the last $500 \mathrm{~Hz}$ of the full frequency range. Usually higher frequency content is expected as you approach the nozzle exit plane. Another issue to consider is the aliasing of higher frequencies in the low frequency bands, which is expected with these optical techniques since there is no way to implement an anti-aliasing filter. Wernet (2007) discussed the effects of aliasing on the TR-PIV data. The same effects can be expected in the Rayleigh data. Aliasing may cause high frequency liftoff in the spectra, resulting in higher turbulence estimates than expected. In the case of the Rayleigh data, the liftoff results in an artificially high noise floor estimate resulting in low turbulence estimates. The lower energy levels exhibited by the Rayleigh technique at $x / D=3$ most likely are the result of over-estimation of the noise floor. Further downstream at $x / D=6$ the Rayleigh and TR-PIV PSDs match up better since the noise floor was more accurately represented by the energy level at $4.5 \mathrm{kHz}$ and beyond. Therefore, it is important to consider the expected fluctuation range when implementing the Rayleigh technique. The radial profiles of rms velocity turbulence in Fig. 8 show that there are some differences in the magnitude of the velocity fluctuations between the three measurement predictions, some of which may be related to inaccurate noise floor estimation and aliasing effects; however the general trend is the same for all three. Although a radial profile like Fig. 8 is useful for direct point-to-point comparisons between the techniques, a contour plot of the flow field produced by fitting a surface function to the grid of measurement points allows for a much easier comparison.

The mean axial velocity normalized by the jet exit velocity for set point 23 is plotted as a function of $x / D$ and $r / D$ for the Rayleigh, TR-PIV, and RANS CFD techniques in Figs. 11a, 11c, and 11e, respectively. The main flow direction is from left to right, with the nozzle exit plane located at $x / D$ $=0$, the centerline of the nozzle at $r / D=0$, and the nozzle lip at $r / D=0.5$. Note that the range of the $r / D$ axis is much smaller than the $x / D$ axis giving a magnified view in the radial direction. The white areas in all of the Rayleigh contour plots indicate areas where data was unavailable. The first two diameters in the PIV data and RANS solutions have been cut off in the figures to make a oneto-one comparison with the Rayleigh data. The TR-PIV data only extends to $r / D=0.675$ in the radial direction. The three velocity predictions are in pretty good agreement, although the Rayleigh data indicates a slightly narrower and shorter potential core than exhibited by the TR-PIV and RANS CFD data. This difference may be related to bias error in the Rayleigh measurements; however the general trend in profile development as the flow progresses downstream is similar for all three techniques. The rms axial velocity fluctuations normalized by the jet exit velocity, expressed as percentage, based on Rayleigh, TR-PIV, and RANS CFD techniques are shown in Figs. 11b, 11d, and 11f, respectively. Again, there are differences in the magnitude of the fluctuations predicted by the three estimation methods but the general trends indicated by the 
curvature of the contours are in good agreement. The magnitude differences between the Rayleigh measurements and the other two methods may be explained by inaccurate noise floor estimates from the PSDs which were used to determine the rms values.

The mean static temperature normalized by the ambient temperature for set point 23 is plotted as a function of $x / D$ and $r / D$ for the Rayleigh and RANS CFD techniques in Figs. 12a and 12c, respectively. Again, the Rayleigh measurements indicate a slightly narrower and shorter potential core compared to the RANS prediction, possibly due to bias error in the Rayleigh technique. The RANS CFD computation provides total temperature fluctuations, whereas the Rayleigh technique measures static temperature fluctuations. Therefore, to make a direct comparison the simultaneous static temperature and axial velocity measurements from the Rayleigh technique are used to derive instantaneous total temperature $T_{o}$ estimates using the following relation:

$$
T_{o}=T_{s t}+\frac{u^{2}}{2 C_{p}}
$$

where $C_{p}$ is the specific heat capacity at constant pressure. The rms total temperature fluctuations are then calculated from the integral of the PSD of the time-resolved total temperature estimates. The rms total temperature normalized by jet exit total temperature, expressed as percentage, based on the Rayleigh and RANS CFD predictions is plotted in Figs. 12b and 12d, respectively. The curvature of the contours is very similar between the two predictions however the Rayleigh technique indicates much higher fluctuation levels. The potential overestimation of the fluctuations may be an artifact of deriving instantaneous total temperature estimates from two very noisy measurements of static temperature and velocity.

The normalized mean axial velocity for set point 46 is plotted for the Rayleigh, conventional $10 \mathrm{~Hz}$ PIV, and RANS CFD techniques in Figs. 13a, 13c, and 13e, respectively. The Rayleigh and PIV data predict a slightly shorter potential core than the CFD prediction. The rms axial velocity fluctuations expressed as a percentage of the jet exit velocity, based on Rayleigh, $10 \mathrm{~Hz} \mathrm{PIV}$, and RANS CFD techniques are plotted in Figs. 13b, 13d, and 13f, respectively. The rms velocity estimates for the $10 \mathrm{~Hz}$ PIV data are obtained by calculating the standard deviation of $200 \mathrm{flow}$ snapshots. The PIV data indicates that the velocity fluctuations are slightly higher than the CFD and Rayleigh predictions. The reason for this discrepancy is unclear. The Rayleigh velocity fluctuations indicate higher levels in the potential core and lower peak levels in the shear layer than predicted by the CFD. The Rayleigh measurements in the core have a high likelihood of being inaccurate since the fluctuation levels are expected to be within the noise level of the technique. The possible underestimation of the fluctuations in the shear layer, especially near the nozzle exit, is most likely due to inaccurate noise floor estimation.

The normalized mean static temperature for set point 46 is plotted for the Rayleigh and RANS CFD techniques in Figs. 14a and 14c, respectively. The Rayleigh measurements slightly overestimate the temperatures right at the nozzle exit, but agree with the CFD in terms of potential core width and length. The rms total temperature fluctuations expressed as a percentage of the jet exit total temperature based on the Rayleigh and RANS CFD predictions are plotted in Figs. 14b and 14d, respectively. The Rayleigh technique overestimates the fluctuations within the potential core due to measurements falling within the noise level of the technique. The Rayleigh technique underestimates the fluctuations on the lip line close to the nozzle exit because the noise floor was predicted to be higher than the true noise floor since the fluctuations had not fully dissipated by the 4.5-5 kHz frequency interval. The Rayleigh technique does a good job of predicting the fluctuation levels in high turbulence regions further downstream of the nozzle exit $(x / D>4)$. 

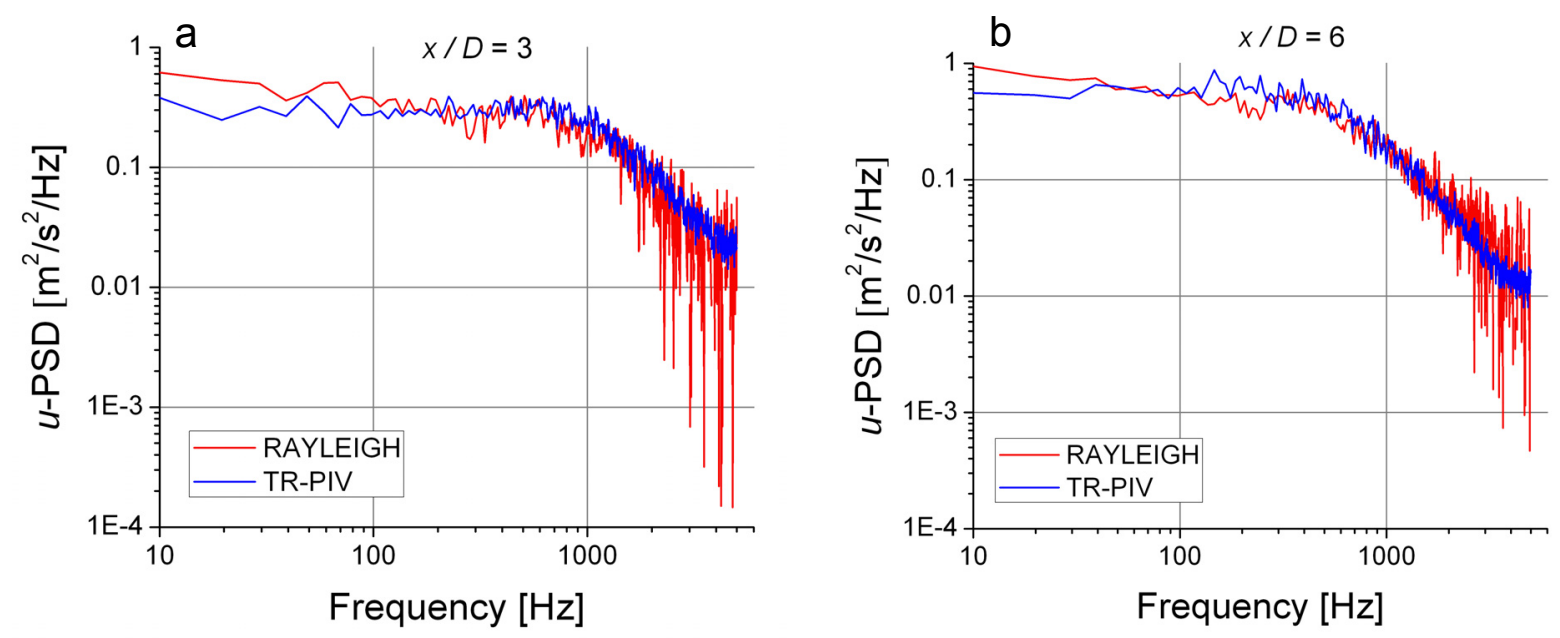

Fig. 10 Axial velocity power spectral densities for set point 23 calculated from $10 \mathrm{kHz}$ Rayleigh and TR-PIV measurements in the shear layer at axial locations of $x / D=3$ (a) and $x / D=6(\mathbf{b})$.
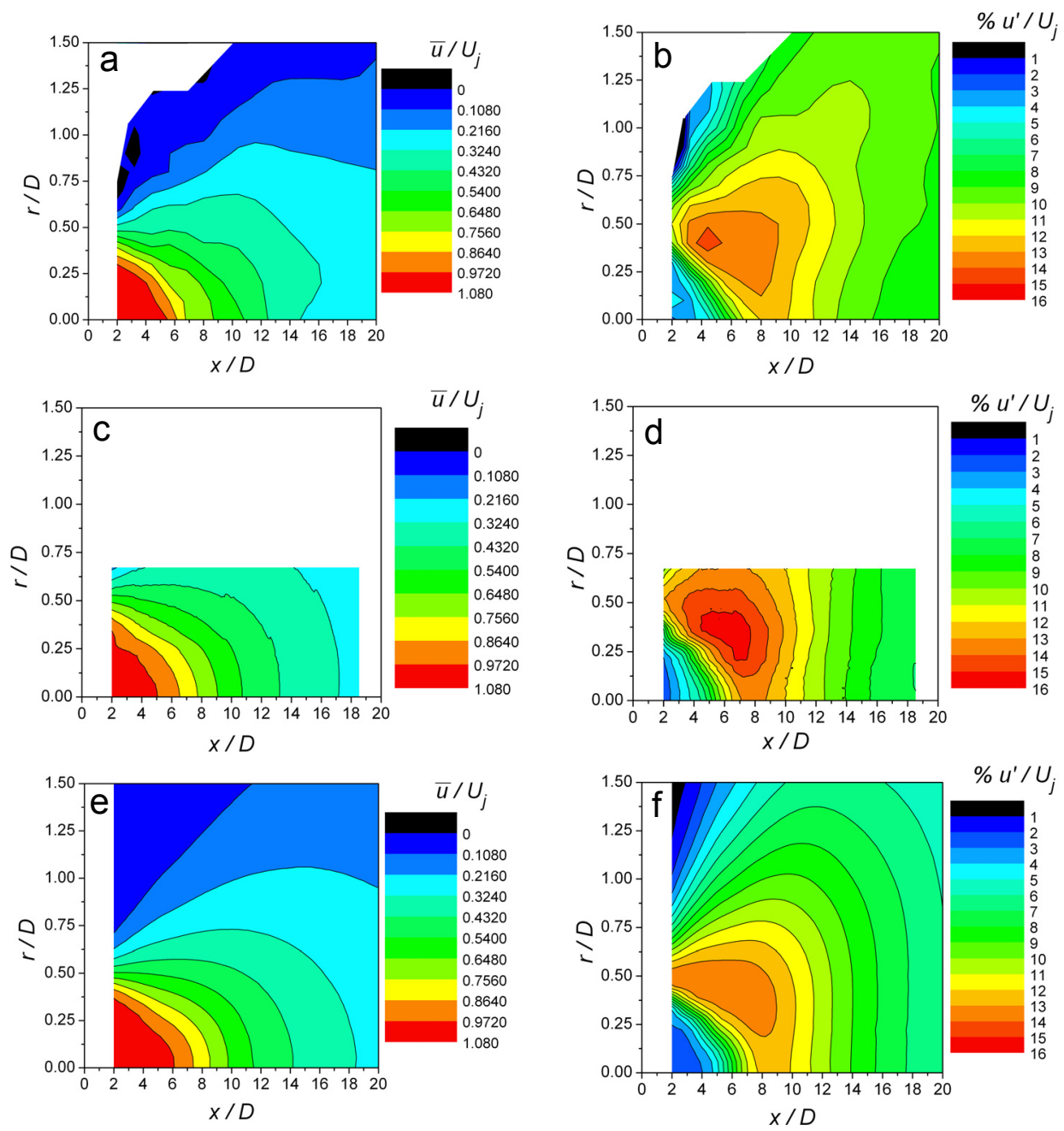

Fig. 11a-f Normalized mean axial velocity (left column) and normalized rms axial velocity fluctuations (right column) for set point 23 based on results from Rayleigh scattering $(\mathbf{a}, \mathbf{b})$, TR-PIV (c, d), and RANS CFD (e, f). 

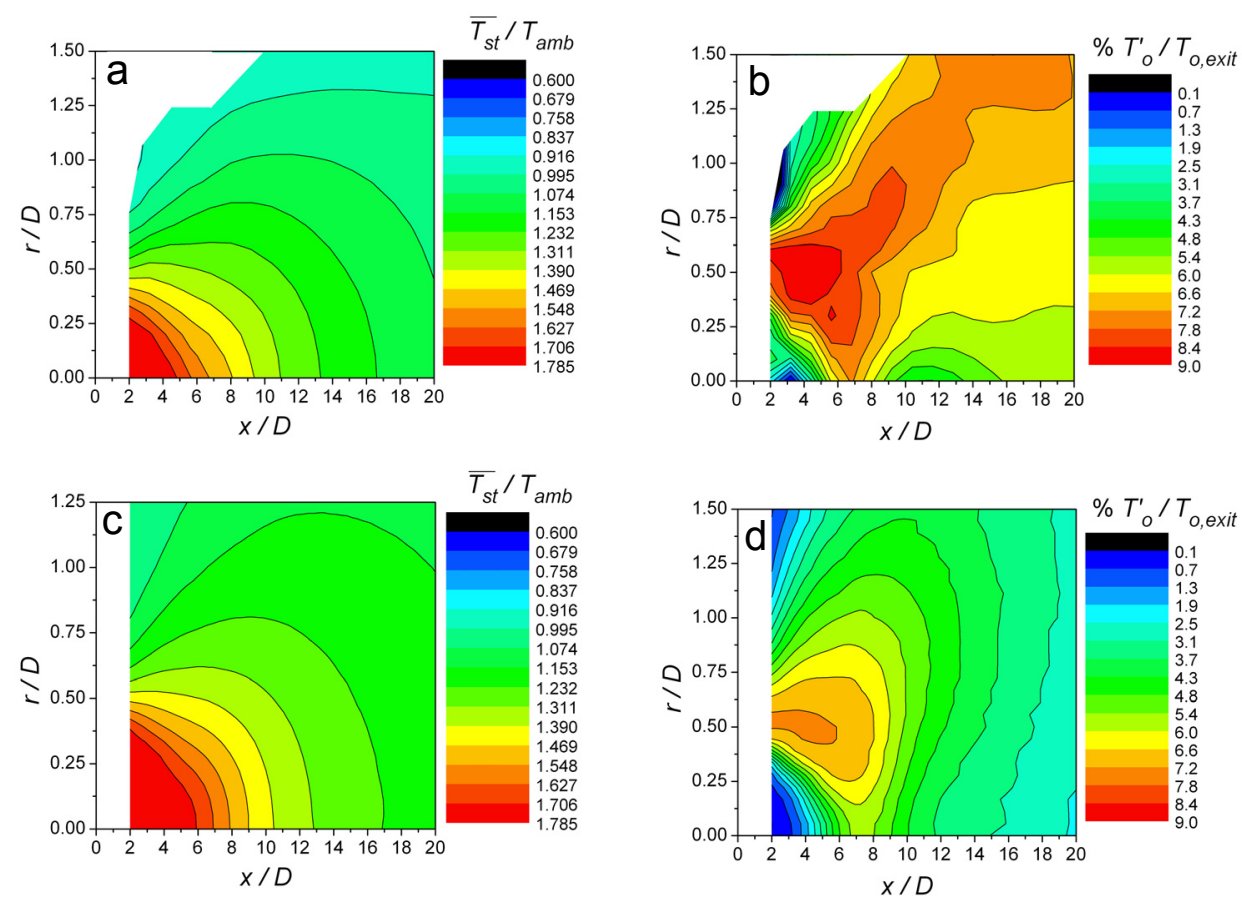

Fig. 12a-d Normalized mean static temperature (left column) and normalized rms total temperature fluctuations (right column) for set point 23 based on results from Rayleigh scattering (a, b) and RANS CFD (c, d).
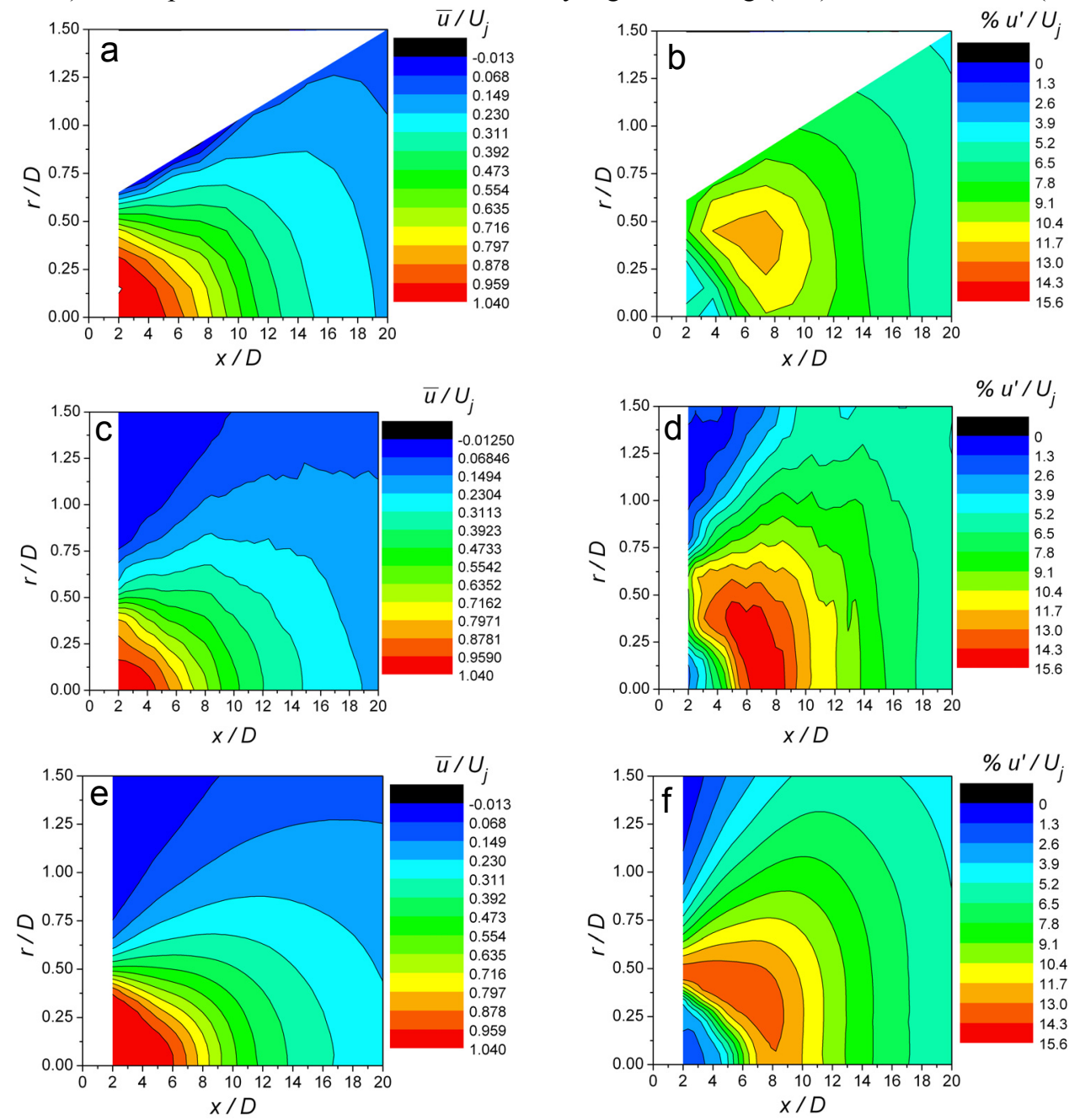

Fig. 13a-f Normalized mean axial velocity (left column) and normalized rms axial velocity fluctuations (right column) for set point 46 based on results from Rayleigh scattering (a, b), $10 \mathrm{~Hz}$ PIV (c, d), and RANS CFD (e, f). 

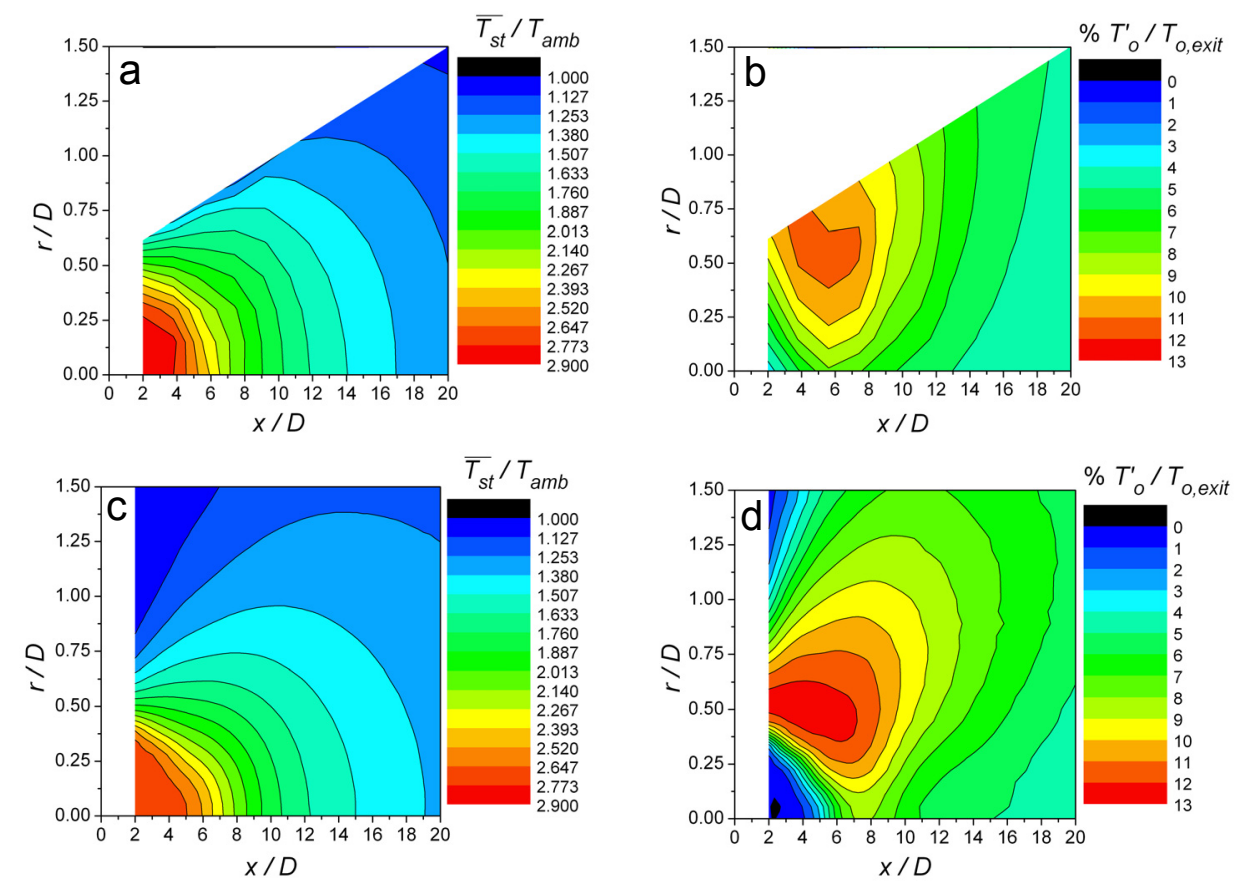

Fig. 14a-d Normalized mean static temperature (left column) and normalized rms total temperature fluctuations (right column) for set point 46 based on results from Rayleigh scattering $(\mathbf{a}, \mathbf{b})$ and RANS CFD $(\mathbf{c}, \mathbf{d})$.

\section{Conclusion}

A technique for obtaining time-resolved gas velocity, temperature, and density measurements using molecular Rayleigh scattering was described. Density was determined from an overall intensity measurement of the scattered light, while temperature and velocity were determined by analyzing the scattered light with a Fabry-Perot interferometer. The signals from five photomultiplier tubes were simultaneously recorded using photon counting at $10 \mathrm{kHz}$ sampling rate. The Rayleigh technique was demonstrated in the Small Hot Jet Acoustic Rig with a 50.8-mm diameter nozzle in NASA GRC's AeroAcoustic Propulstion Laboratory. Mean and rms velocity and temperature measurements obtained by Rayleigh scattering were compared to TR-PIV and conventional PIV measurements as well as RANS CFD predictions. The root mean square fluctuations and power spectra were obtained with increased accuracy by utilizing noise floor subtraction. The Rayleigh technique is limited by the accuracy of estimating the shot noise floor. Improved methods of noise floor estimation using numerical simulation prediction are being investigated to increase the accuracy of the Rayleigh rms turbulence estimates. The complete set of data acquired in the SHJAR will be analyzed and presented in a later publication to provide an experimental database for validation of computational codes.

\section{Acknowledgments}

The authors would like to thank Dennis Eck of Jacobs Sverdrup and Ray Loew of Sierra Lobo for their support in the setup and operation of the SHJAR facility and also Pete Eichele of Gilcrest for his assistance in the setup and installation of the Rayleigh scattering system. We would also like to thank Mark Wernet of the Optical Instrumentation and NDE Branch and James Bridges of the Acoustics Branch at NASA GRC for providing the PIV data that was presented, Abbas Khavaran and Donald Kenzakowski for providing the RANS CFD predictions, and G. Tenti of the University of Waterloo for providing the 6-moment Rayleigh spectrum calculation code. 


\section{References}

Boguszko M, Elliott G S (2005) On the Use of Filtered Rayleigh Scattering for Measurements in Compressible Flows and Thermal Fields. Exp Fluids 38:33-49

Boley C D, Desai R C, Tenti G (1972) Kinetic Models and Brillouin Scattering in a Molecular Gas. Can J Phys 50:2158-2173

Bonnet J P, Grésillon D, Cabrit B, Frolov V (1995) Collective Light Scattering as Non-Particle Laser Velocimetry. Meas Sci Technol 6:620-636

Bridges J, Wernet M P (2003) Measurements of the Aeroacoustic Sound Source in Hot Jets. AIAA-20033130

Cummings E B (1995) Laser-Induced Thermal Acoustics. PhD Dissertation, California Institute of Technology, Pasadena, California

Eckbreth A C (1996) Laser Diagnostics for Combustion Temperature and Species. Gordon and Breach Science Publishers SA, Amsterdam, pp 209-451

Hart R C, Balla R J, Herring G C (1999) Nonresonant Referenced Laser-Induced Acoustics Thermometry in Air. Appl Opt 38:577-584

Kenzakowski D C (2006) RANS Modeling Improvements for Jets Using Scalar Variance Equations. AIAA2006-0491

Khavaran A, Kenzakowski D C (2007a) Progress toward Improving Jet Noise Prediction in Hot Jets. AIAA2007-0012

Khavaran A, Kenzakowski D C (2007b) Noise Generation in Hot Jets. AIAA-2007-3640

Koochesfahani M M (1999) Molecular Tagging Velocimetry (MTV): Progress and Applications. AIAA-993786

Lock J A, Seasholtz R G, John W T (1992) Rayleigh-Brillouin Scattering to Determine One-Dimensional Temperature and Number Density Profiles of a Gas Flow Field. Appl Opt 31:2839-2848

Mielke A F (2008) Development of a Molecular Rayleigh Scattering Diagnostic for Simultaneous TimeResolved Measurement of Temperature, Velocity, and Density. Ph.D. Dissertation, Case Western Reserve Univ, Cleveland, Ohio

Mielke A F, Elam K A, Sung C J (2008) Time-Resolved Rayleigh Scattering Measurements in Hot Gas Flows. AIAA-2008-262

Mielke A F, Seasholtz R G, Elam K A, Panda J (2005) Time-average Measurement of Velocity, Density, Temperature, and Turbulence Velocity Fluctuations Using Rayleigh and Mie Scattering. Exp Fluids 39:441-454

Nagano Y, Kim C (1988) A Two-Equation Model for Heat Transport in Wall Turbulent Shear Flows. J Heat Transfer 110:583-589

Panda J, Seasholtz R G (1999) Velocity and Temperature Measurement in Supersonic Free Jets Using Spectrally Resolved Rayleigh Scattering. AIAA-99-0296

Panda J, Seasholtz R G, Elam K A, Mielke A F, Eck D G (2004) Effect of Heating on Turbulent Density Fluctuations and Noise Generation from High Speed Jets. AIAA-2004-3016

Seasholtz R G, Panda J, Elam K A (2002) Rayleigh Scattering Diagnostic for Measurement of Velocity and Density Fluctuation Spectra. AIAA-2002-0827

Tenti G, Boley C, D, Desai R C (1974) On the Kinetic Model Description of Rayleigh-Brillouin Scattering from Molecular Gases. Can J Phys 52:285-290

Vaughan J M (1989) The Fabry Perot Interferometer, History, Theory, Practice, and Applications. Adam Hilger, Philadelphia, pp 89-134

Welch P D (1967) The Use of Fast Fourier Transform for the Estimation of Power Spectra: A Method Based on Time Averaging Over, Short Modified Periodograms. IEEE Trans Audio Electroacoust AU-15:70-73

Wernet M P (2007) Temporally Resolved PIV for Space-Time Correlations in Both Cold and Hot Jet Flows. Meas Sci Technol 18:1387-1403 